\title{
1 \\ The Welfare of Animals and Its Relevance to Our Health
}

We must fight against the unconscious cruelty with which we treat animals ... Until he extends the circle of compassion to all living things, man will not himself find peace.

-Dr Albert Schweitzer

\section{Our common fate}

Whether humans will ever find peace is up for conjecture, but this book aims to demonstrate that until we improve the welfare of non-human animals, we will never find health. For many involved in the health field, this proclamation will come as a great surprise. For others, it might be viewed as approaching heresy. How could the medical field, which is charged with the enormous responsibility of promoting human health and alleviating our suffering, also be concerned about the welfare of animals? It may be argued that animal welfare has nothing to do with human health, or even, more broadly, with human welfare. Yet, the notion that the way in which we treat animals impacts our own welfare is not a new one. Philosophers, scientists and other thinkers, dating from ancient Greece to modern times, have long suggested that when we disregard the welfare of other animals it may come back to haunt us in one way or another. The list of such thinkers is long and includes distinguished names such as Pythagoras, Plutarch, Socrates, Albert Einstein, St Francis of Assisi, Jean Jacques Rousseau, Immanuel Kant and Isaac Bashevis Singer.

Much of these earlier reflections were speculation, but today there is mounting evidence of a very real, and often very direct, relationship between animal welfare and human welfare, most specifically with regard to human health. While this book will focus mostly on human 
health, other aspects of human welfare (such as freedom from violence, crime and hunger) that have been connected with human health and animal welfare will also be explored. For instance, as shown by the opening quote, Schweitzer suggested that the poor treatment of animals can result in the inability of humans to find peace. Moreover, a connection has been made between cruelty toward animals and violence toward humans. This book will demonstrate how our health can be greatly influenced, positively or negatively, by how we choose to treat animals. By taking a look at some recent, highly publicized events that have threatened human health and welfare, a better picture of how human health and animal welfare are connected can be formed.

For instance, Chapter 4 will describe how, in 2003, avian influenza (H5N1 strain) spread swiftly across poultry farms in Asia and jumped the species barrier to infect humans, raising red flags that the next pandemic could originate from animal farms. ${ }^{1}$ When, in 2009, swine (H1N1 strain) influenza swept across the globe, it was confirmed that animal agriculture can play a significant role in the emergence of new strains of influenza viruses. Animals, living in profoundly filthy and crowded conditions that severely compromise their welfare and their immune systems, are now predominantly raised for food in 'factory farms' or confined animal-feeding operation. This creates perfect breeding grounds for new infectious diseases that are potentially deadlier than those already witnessed.

Another way in which human health and animal welfare are connected can be seen in the responses of people during Hurricane Katrina in 2005. The world watched live television as many Louisiana residents refused to evacuate their homes in the wake of the hurricane and, in some cases, risked death to avoid losing their companion animals (who were not permitted on Coast Guard rescue vehicles or welcome in local shelters). Some 44 percent of those who refused to evacuate did so because they did not want to leave their animals behind. ${ }^{2}$ Indeed, the most common reason people return to evacuation sites is to rescue their pets. ${ }^{3}$ Post-Katrina studies show that the loss of these companions worsened the mental trauma many people experienced. ${ }^{4}$ This was a wake-up call for public health and rescue agencies throughout the world to take the human-animal bond seriously and incorporate animal rescue into emergency plans, since not doing so puts human health and welfare at risk (not to mention the health and welfare of the companion animals in question).

In 2007, a worldwide recall of pet food that had been exported from China and was contaminated with melamine was ordered after possibly thousands of animals fell ill or died. ${ }^{5}$ In addition to the public 
outrage this prompted, there was a perceived human risk since some of the tainted pet food was also fed to animals later processed into human food. Because of this incident, the need for improved regulatory monitoring of food fed to animals, so as to protect both humans and animal companions, became evident. ${ }^{6}$

In 2009, a woman in Connecticut, USA, suffered a dreadful attack by a pet chimpanzee; and in 2010, SeaWorld animal trainer Dawn Brancheau was killed by an orca in front of a horrified audience. These events underscored the dangers of using wild animals for entertainment and as pets. ${ }^{7}$ No one knows for certain why these animals attacked but, as explored in Chapter 3, wild animals raised as pets or used for entertainment are often kept in deplorable or inadequate housing conditions, deprived of any semblance of a natural life and may be subjected to other forms of abuse. Although a direct link between these conditions and such attacks can be difficult to establish, having wild animals in close proximity to humans certainly increases the opportunities for such attacks and can expose people to novel infectious diseases, posing immense public health risks.

Arguably, one of the most significant and pressing public health issues of our time is climate change. Reports from the United Nations' Food and Agriculture Organization in 2006 and the Pew Charitable Trust in 2008 have increased awareness and acknowledgment of the connection between what we eat and climate change. ${ }^{8}$ Chapter 5 describes how the unprecedented worldwide demand for meat and the subsequent rise in factory farming affect our climate in significant ways. Modern animal agricultural practices contribute more to greenhouse gas emissions and environmental degradation than many of the industries on which we have traditionally pinned the blame.

These examples all illustrate the important connection between human health and animal welfare. As will be demonstrated throughout this book, when we treat other animals well, there are clear direct and indirect benefits to human health. When we treat animals poorly, our collective health suffers. Chapter 2 will demonstrate how the abuse of animals is correlated with the abuse of humans, particularly women and children. In Chapter 3 we will explore how our shipment of animals around the globe for food, fur, the purposes of entertainment and the acquisition of exotic pets is directly and indirectly linked to some of the most dangerous epidemics we have faced in recent decades. Chapters 4 and 5 explore how factory farms are polluting our land, water and air and are making us ill. Chapter 6 presents mounting evidence demonstrating that the use of animals in experimentation is unreliable, inefficient and dangerous to human health. 
On the positive side, as Chapter 6 further explores, when we strive to improve the welfare of animals by reducing their numbers in experimentation and replacing their use with non-animal research methods, we discover testing methods that more successfully predict human reactions to toxins and drugs. These human-based testing methods are proving to be better at protecting people from harmful chemicals than animal experiments, as well as providing the answers we need to find effective treatments and cures for the diseases that ail us. As Chapters 4 and 5 show, reducing our consumption of animals would confer great personal health benefits by substantially decreasing our risks of strokes, diabetes and cancers, and by helping us live longer, healthier lives. Additionally, it would decrease the number of factory farms and thus the number of animals living in such conditions. This in turn would help us avoid pandemics and limit further destruction of our environment.

Despite the link between animal welfare and human health, animal welfare issues have been, with few exceptions, notoriously absent from the public health dialog. When the subject of animals does enter discourse on human health, it is usually to highlight how animals are sources of infection for and cause injuries to humans. There has been almost no discourse, however, about the fact that the way in which we treat other animals is often central to how and why humans are injured or catch infections. Moreover, such treatment is central to how and why we face a significant number of health threats. This distinction is important to note. To illustrate this, it helps to take a look at how the public health discipline has traditionally approached the subject of zoonosesinfectious diseases that can be transmitted from non-human animals to humans (and vice versa). There is ample medical literature exploring the variety of zoonoses that can be passed from animals to humans. There is also much medical literature and many studies exploring how and why we contract zoonoses and pass them on to each other. Such work looks predominantly at human-human interactions, weather patterns and the life cycle of infectious agents. When public health practitioners and investigators do turn their attention specifically to animals, it is usually to describe which animals are likely to be infected and thus pose a threat to us. Rarely do we explore deeper than this and ponder whether the nature of our relationships with animals could play a role in whether they become infected with a pathogen in the first place or pass the pathogen on to us.

When human infection from a zoonotic disease grows to epidemic proportions, the public health response is frequently swift and 
unforgiving: we simply kill or 'cull' animals in hopes of thwarting a pandemic. When, in 2003, severe acute respiratory syndrome (SARS) swept across the globe, civet cats sold at wet markets (open markets selling live animals) in China were found to be infected with the SARS coronavirus. Civets are one of the thousands of species bred in captivity or captured from their natural habitats to be sold for exotic meat, fur, traditional medicines and other commercial purposes in the massive worldwide wildlife trade. Upon discovering the SARS coronavirus in civets, China's public health response was to kill thousands of these and other animals by drowning them in disinfectants and electrocuting and poisoning them. ${ }^{9}$ In addition to killing, other tactics involved dosing animals with massive amounts of vaccines or antimicrobials, which creates the potential to cause additional problems, such as antimicrobial resistance.

After China's mass execution of civets, research later revealed that bats, not civets, are the likely primary source of SARS. But we should not rush to a judgment on bats either. Bats, as well as civet cats, are victims of the wildlife trade, which, as argued in Chapter 3, is the true culprit. As explored there, both the wildlife trade and wet markets are guilty of deplorable and inhumane conditions in which animals are kept and, frequently, killed. Because of these conditions, animals in the wildlife trade easily experience compromised immune systems, which render them highly susceptible to contracting pathogens from other animals. Evidence points to the wildlife trade and the live markets as being the nexus in which the SARS coronavirus passed from bats to other animals, including civets and, ultimately, humans.

Undeniably, pathogens transferred from animals to humans have led to human epidemics, but rarely do we ask whether the way we treat animals could have played a role in the epidemics, and whether better treatment might have prevented them from beginning in the first place. ${ }^{10}$ By ignoring the welfare of animals, we fail to see the most direct and comprehensive (and sometimes the simplest) solutions. In the case of SARS, such an epidemic might never have occurred if we had treated animals better. If we had considered the welfare of animals, we would likely have placed greater restrictions on their sale in wet markets and for the wildlife trade. The reduction in, or elimination of, these practices would have reduced our chances of contracting a pathogen from animals. At the very least, if we had improved their conditions, perhaps fewer animals would have contracted SARS and passed it on to us. 
Undoubtedly, treating other animals better is not the solution to every public health problem we face and, of course, no matter how well we treat animals, they may still become infected and end up being associated with the next major public health calamity. But we are being remiss, and arguably grossly negligent, when we avoid discussing our treatment of other animals as a potential cause and potential solution altogether. A considerable amount of human suffering may be avoided if, rather than asking ourselves how to thwart an epidemic once it has begun, we instead ask ourselves whether we can prevent an epidemic by treating animals differently.

The consideration that our treatment of non-human animals impacts human health and disease notwithstanding, there are several objections that can be raised when considering the welfare of animals and its link with human health. The first potential argument against considering animal welfare is that it makes no sense to discuss their welfare because, in particular, animals don't feel pain or suffer (there are other potential components to their welfare, such as living in freedom or being among their own kind, which are touched upon briefly in this book). Thus, as this argument goes, because animals are incapable of feeling pain and of suffering, their welfare is a non-issue. Alternatively, one may acknowledge that animals can and do feel pain and perhaps suffer, but that their potential for these is extremely limited or fundamentally different from that of humans. Again, with respect to pain and suffering, some may argue that the welfare of animals is a non-issue or, at best, a minor issue. The second objection is, assuming that one acknowledges that animals can feel pain and suffer, that human health nevertheless takes priority over the welfare of animals. The third objection, an extension of the second, is that animals must be utilized in ways that may cause their suffering in order to promote human health. Finally, the last major objection is that animal welfare is a social issue that is outside the purview of public health. Each of these objections is addressed in the following sections.

\section{Pain and suffering}

Do animals feel pain and suffer? It is very tempting to bypass this question and assume that the reader, as most people, including the general public and scientific experts, acknowledges that all mammals and most vertebrate animals can experience pain and can suffer. However, there are still those who argue that animals cannot experience pain and/or suffering and who use that assertion as a justification to deny the 
legitimacy of animal welfare and defend some of our current treatment of animals.

The terms 'pain' and 'suffering' are often used interchangeably, but there is widespread debate as to what suffering actually means and discussion of how certain forms of suffering may be distinct from the experience of pain. Although this is a complex topic that others have written extensively about, a few of the more relevant points can be addressed here. First is the question of pain. Pain is usually taken to mean a negative sensation in response to adverse or noxious stimuli. The majority of people today, including philosophers, animal behaviorists, physiologists and other scientists, acknowledge that vertebrate animals feel pain. In addition, substantial scientific evidence demonstrates that most, if not all, vertebrates, including all mammals, birds, reptiles and fish, as well as many invertebrates, such as cephalopods and crustaceans, feel pain. ${ }^{11}$

Vertebrate animals display nearly all the same external signs to an unpleasant stimulus as humans: they writhe, cry out (some within our own range of sounds and some ultrasonically), yelp, show facial contortions, avoid putting weight on an injured limb, demonstrate decreased appetite and interrupt their normal behaviors. These external signs distinguish most animals from other organisms, such as an amoeba, which may avoid a harmful stimulus but demonstrate no other manifestation that suggests an experience of pain. Animals also show characteristic physiologic reactions suggestive of pain. Like humans, they show elevated respiration and heart rate, dilated pupils, release of stress hormones (e.g. cortisol and norepinephrine), and they often respond to pain-alleviating medications in a similar fashion to humans. Even fish, when subjected to chemical irritants, demonstrate a response to morphine, suggesting that they experience pain and that the drug can alleviate it. ${ }^{12}$ We can also infer animals' pain by the structural similarities of their nervous systems to our own. While inter-species differences exist, all vertebrates evolved to share many of the basic neurophysiological features that allow for the processing and perception of pain. For example, nociceptors (pain receptors) are widespread in the tissues of a wide range of animals, and chemical modulators of these receptors, such as endogenous opioids, exist in both humans and non-human vertebrates-and at least in some invertebrate animals. Thus, the weight of evidence points to animals (at least the animals discussed in this book) as being capable of experiencing pain. With respect to the topics covered here, since much of our treatment of animals involves causing them pain, animal welfare deserves serious attention. 
The second question concerns the topic of suffering. There are many philosophers and scientists who maintain there are forms of suffering that are distinct from pain and, moreover, that there are many different concepts and criteria of suffering. ${ }^{13}$ Pain can be one component of suffering, but it is not a necessary component. Common forms of suffering that do not necessarily involve pain include negative cognitive or psychological experiences, such as the experience of fear, anxiety, distress, emotional trauma, foreboding, loneliness and terror. ${ }^{14}$ For example, if a woman experiences a stroke and becomes paralyzed on one side of her body, she may suffer from the stroke even if no pain is involved. She may suffer because she is unable to perform what were previously normal functions for her and she may experience distress and anxiety as a result of her limited function.

Pain can also cause limited function, which can lead to negative emotional or cognitive experiences. An individual with an injured leg may be limited in how he or she uses that leg because of the pain that may follow. This limited function may cause distress and immense frustration, which can be forms of suffering apart from the experience of pain itself. It has been argued that animals lack cognitive and emotional capabilities to experience distress, terror, anxiety, loneliness and the like, and thus cannot suffer in these ways, or they suffer much less in comparison with humans. But is it true that animals lack these abilities? This next section will briefly present some of the amounting evidence that shows animals have a wide range of emotional and cognitive capabilities and are therefore likely to suffer-at least in terms of their ability to experience negative emotional and/or psychological states, such as distress, terror and loneliness.

The evidence that animals can suffer from negative emotional and cognitive states is accumulating, in part, because of a revolution in the field of animal behavior, which is breaking the long-held taboo against studying animal minds. ${ }^{15}$ There is now an explosion of work by notable scientists, such as Frans de Waal, Michael Tomasello, Marc Bekoff, Sue Savage-Rumbaugh and Jaak Panksepp, examining the mental lives of animals (how they think and feel). These studies reveal that many animals experience a range of emotional and cognitive capacities that were previously denied to them. In what follows, a brief array of evidence from such studies will be presented to indicate these capacities. The evidence is such that it is extremely difficult to deny that animals are incapable of suffering, and it shifts the burden of proof from having to show that animals suffer to having to show that animals do not. 


\section{Language and cognitive abilities}

Many have heard of the famous chimpanzee, Washoe, who was the first non-human to learn parts (albeit truncated) of American Sign Language. What might surprise some, however, is that she passed on a portion of her sign language knowledge to her adopted chimpanzee son, Loulis, without any inducement from humans. ${ }^{16}$ Stimulated by the success of Washoe's language abilities, other investigators achieved similar levels of communication with other chimpanzees, gorillas and orangutans. ${ }^{17}$ Orangutans have learned to communicate with humans using computer touch screens. ${ }^{18}$ The communication skills of the great apes have many scientists arguing that they do indeed possess the capacity for some form of language, the primary attribute that some believe separates humans from all other animals. ${ }^{19}$ Chimpanzees also show evidence of spontaneous acts of kindness, social awareness and culture. ${ }^{20}$ From such evidence, some have argued that they exhibit 'theory of mind' - that is, the ability to attribute beliefs, desires and other reference-making abilities to other beings, be they human or chimpanzee, and that they rely on such attributions to inform and shape their interactions. ${ }^{21}$

Tufted capuchin monkeys, a New World primate, recognize photographs of faces of familiar monkeys and can pick out photos of individuals who do not belong to their social groups. ${ }^{22}$ Studies suggest that rhesus monkeys know when they know something and know when they don't. In a series of experiments, researchers found that these monkeys chose to take only memory tests on which they were found to perform well and declined to take tests they would likely fail. ${ }^{23}$ This suggests that the monkeys could gauge the apparent difficulty of a test or knew whether or not they remembered the correct responses. This ability to make adaptive decisions about future behavior contingent on the current availability of knowledge is a capacity associated with cognition in humans. ${ }^{24}$

\section{Emotional and other abilities}

Given their close genetic relationship with us, it's not surprising to discover the nuanced cognitive traits in non-human primates. But what might surprise many is that even the 'lowliest' of animals— the ones who throughout history we have most disregarded-are far more complex than we commonly believe. To take an example, studies suggest that rats and mice experience empathy. In one famous, yet lamentable, experiment performed in the 1950s, psychologist Russell Church trained rats 
to obtain food by pressing a lever. ${ }^{25}$ He found that if these rats saw that, by pressing the lever, another rat in a neighboring cage would receive an electric shock, the first rat would interrupt her activity. A more recent and highly publicized (and also not so nice) study reveals a potential form of empathy in mice. ${ }^{26}$ Here, researchers injected painful irritants into mice, then watched them writhe in pain. When other mice witnessed this, they showed heightened sensitivity to pain themselves. But the observer mice mostly only reacted to the pain being experienced by other mice with whom they were familiar. This strongly suggests that the responses of the mice were more than simply an automatic fear reaction and that they were selective to specific mice- $\mathrm{a}$ form of empathy. In a review of empathy studies in mice and rats, leading animal behaviorist Frans de Waal stated,

Apart from a few rear-guard behaviorists, few people hesitate to ascribe empathy to their dogs. But then dog is man's best friend, freely credited with lots of human sentiments. You wouldn't expect a hard-nosed scientist to make similar claims about, say, rodents, would you? Yet a significant line of research ... demonstrates not just empathy's existence in rodents and other animals but its subtleties and exceptions as well. ${ }^{27}$

Tickle a rat's belly and he will emit ultrasonic chirping sounds, which are believed to have the same neural basis as human laughter. ${ }^{28}$

In the 1980s Gerald Wilkinson made a significant discovery about vampire bats: they are altruistic. These bats feed on the blood of larger mammals. Food sources can be scarce so that they may go for long periods of time without food. If a bat is starving, other bats will regurgitate blood to share with him or her, compromising their own nourishment. ${ }^{29}$ Hens display nuanced communication with other hens. ${ }^{30}$ For example, they use specific calls to relay detailed information about food to other hens. ${ }^{31}$ They finesse their calls when relaying to others that a found food is particularly preferable. Chickens demonstrate the ability to recognize each other by their facial features. ${ }^{32}$ Several studies also suggest that they have the ability to understand that an object, when taken away and hidden, continues to exist - $\mathrm{a}$ feat beyond the capacity of very young children. ${ }^{33}$ Jay birds use deceptive tactics to hide food when they know other birds are watching them. ${ }^{34}$ What is perhaps more interesting, however, is that such deception most frequently occurs when the jay birds hiding the food have previously stolen food from other birds. This suggests that they make assumptions about the actions of other birds based 
on their own. In other words, it takes a thief to know one. Scrub jays also display memory, and an awareness of the passage of time and its effect on different foods. Pigs can show a pessimistic or optimistic outlook on life, depending on whether their environment is dull or stimulating. ${ }^{35}$ And a recent study found that domestic pigs quickly learn how mirrors work and will use their understanding of reflected images to scope out their surroundings and find their food. ${ }^{36}$

Reptiles are commonly thought of as being unemotional creatures. However, physiologist Michael Cabanac demonstrated an emotional fever response in lizards and turtles resulting from human handling. ${ }^{37}$ This is akin to studies which demonstrate that in rats body temperatures rose by $1{ }^{\circ} \mathrm{C}$ or more when handled by unfamiliar persons. This response disappears when the same person handles the rat repeatedly over several days, as the rat develops trust in the handler. If another handler is introduced, the emotion-caused fever returns. The similarity in the fever response between rats and reptiles suggests that reptiles also experience fear or distress, which can manifest as a rise in body temperature.

\section{Continuity with humans}

These are just a few of the many examples of studies revealing animal emotion and cognition. Given the evolutionary and biological continuity across species, it is more and more difficult to claim that there exists any strong dichotomy between humans and other animals, at least when it comes to basic emotional and cognitive abilities that can give rise to suffering. ${ }^{38}$ Eminent psychologist and Senior Fellow in Theoretical Neurosciences at the Neurosciences Institute in California, Bernard Baars, summed up the evidence rather nicely:

All animals engage in purposeful action... other animals investigate novel and interesting stimuli as we do, ignore old and uninteresting events just as we do... Human beings are different of course... But we can no longer pose absolute barriers between ourselves and other animals ... ${ }^{39}$

Granted, many of the studies on animal minds are preliminary and there are other studies that may contradict some of the above findings. Far more needs to be done to better delineate which capabilities exist and in which species. At the very least, however, these initial studies suggest that some emotional and cognitive capabilities previously attributed 
only to humans are more widespread than previously thought, even if many of these capabilities may be restricted in other species as compared with humans. And, as we devise more sophisticated (and kinder) ways to study animal minds in their natural environments and contexts, we will likely discover that they posses many more capacities than we currently assume. ${ }^{40}$ As Frans de Waal stated, 'efforts to single out distinctly human capacities have rarely held up to scientific scrutiny for more than a decade, such as claims about culture, imitation, planning, and the ability to adopt another's point of view ${ }^{\prime 41}$ Even aquatic animals, such as whales and dolphins, who are quite removed from humans on an evolutionary scale have surprised us with their abilities. Carl Sagan's famous tongue-in-cheek quote is a humorous reminder of some of the capabilities which exist in such animals: 'while some dolphins are reported to have learned English-up to fifty words used in correct context-no human being has been reported to have learned dolphinese'.

The notion that we may share some basic cognitive capabilities with other animals makes evolutionary and biological sense. ${ }^{42}$ As the scientific evidence demonstrates, animals are not mere automata, living solely off instinct; they display every indication of being able to feel pain and of having emotional and cognitive abilities that rely on more than mere stimulus-response behaviors. In other words, science shows that animals have mental lives and that their actions are purposeful.

In fact, our very use of animals in experimentation is often predicated on these abilities. According to Colorado State University bioethicist and Professor of Animal Sciences Bernard Rollin, 'not only does much scientific activity presuppose animal pain as we have seen vis-à-vis pain research and psychological research, it fits better with neurophysiology and evolutionary theory to believe that animals have mental experiences than to deny it'. ${ }^{43}$ For instance, many of the medical experiments conducted on animals to understand human disease, particularly psychological experiments, reveal just how much science as an institution agrees that animals experience pain and have various cognitive and emotional abilities. Scientists have intentionally caused and studied the effects of chronic depression, post-traumatic stress disorder, obsessive-compulsive disorder, severe anxiety, schizophrenia and dementia in animals, including mice and rats. The scientific community often attempts to distinguish what animals experience from human experiences by referring to the psychological states induced in the laboratory in a type of scientific doublespeak. For example, animals in 
laboratories aren't routinely described in the biomedical literature as experiencing depression but as displaying depression-like signs.

Yet, if the scientific community did not believe that animals were capable of experiencing these complex psychological states and conditions, why would these studies be conducted in the first place? If the scientific community did not believe that animals experience depression, why would studies on drugs intended to treat depression be conducted on animals? If the scientific community did not believe that animals possess at least some cognitive abilities, why would studies on the loss of cognitive abilities (for research in dementia) be conducted in animals? It stands to reason that the scientific community operates on the assumption that animals possess a wide range of emotional and cognitive capacities. And it makes sense to believe this: the animals in these studies display signs remarkably similar to those seen in human forms of these psychological illnesses or conditions. If these animals exhibit these signs in fairly systematic ways, then, in conjunction with their neurophysiological similarities and evolutionary continuity, it is a natural and scientifically sound inference to conclude that they experience chronic depression and other emotional and psychological states. In other words, animals don't just show distress, they feel it.

Further, if these animals experience depression, anxiety and distress, then it is plausible that they are also suffering in these circumstances. These experiences certainly count as suffering in humans and there is not any scientifically sound reason to believe that this is not also the case in animals. In fact, the US Public Health Service Policy on Humane Care and Use of Laboratory Animals reflects the implicit scientific acceptance of these psychological states in animals and their ability to suffer by suggesting that researchers 'limit the use of animals or limit animal distress' and that 'investigators should consider that procedures that cause pain or distress in humans beings may cause pain or distress in other animals'. ${ }^{4}$

There will be those who will take what has just been stated as evidence that animals are effective tools for medical research. They may contend that if humans and animals share some of the same basic neurophysiological processes and cognitive and emotional abilities, then this implies that animals make good models for human disease. But this argument would be missing a vital element. Medicine now deals with the subtle nuances of physiological mechanisms in order to precisely target an intervention, such as a drug to boost or inhibit a specific cellular or genetic process. The more we study other animals, the more we learn 
how species-and even strains within a species-differ in these subtle mechanisms. It is specifically these differences that render the reliability of animal experiments to predict human outcomes highly questionable. This topic is further explored in Chapter 6.

\section{Summary of suffering}

As animal behaviorist Marc Bekoff states, 'we must be careful neither to imbue animals with unknown cognitive capacities nor to rob them of skills they might possess' ${ }^{45}$ No doubt species differ in their cognitive and emotional capabilities and there are forms of suffering that are likely to be unique to humans. A dog may not suffer from knowledge that he may not meet his grandchildren, though a human might. But even if animals sometimes suffer differently from humans, why should that matter? An animal's experience does not need to be identical to a human's for it to count. Consider color perception as an example. Goldfish, Japanese dace fish, turtles and many birds are tetrachromats-that is, their retinas contain four classes of cone cells for picking up information from wavelengths of light in the red, green, blue and ultraviolet parts of the color spectrum. ${ }^{46}$ There is fairly good evidence that pigeons and ducks may even be pentachromats (possessing five channels or cone cells for conveying color information) ${ }^{47}$

In contrast, normal humans are trichromats, with three classes of cone cells picking up information from the red, green and blue parts of the color spectrum. And about 1 percent of men are dichromats, only having the ability to pick up information from blue and either red or green wavelengths of light. Because of this difference, tetrachromats can rely on a broader range of information to see the world than we do. Tetrachromatic hummingbirds, for instance, can distinguish nearultraviolet light $(370 \mathrm{~nm})$ both from what appears to us as darkness and from 'white' light lacking wavelengths below $400 \mathrm{~nm} .{ }^{48}$ Humans cannot perform either of these tasks. Does the fact that humans perceive color differently, and in a more limited way, than hummingbirds imply that humans don't see color? Of course not; we just process color information differently. The same analogy can be applied to the question of animal cognition and the capacity of animals to suffer. Even if animals do not suffer in all the same ways as humans, this does not mean they don't suffer. We may also want to acknowledge that there may be forms of suffering that are unique to other species. If we destroyed a hummingbird's ability to perceive ultraviolet light, this might lead to a form of suffering unimaginable to humans. By destroying a hummingbird's 
ultraviolet perception, a function that is normal for her, we might be causing that bird to suffer even if we did nothing else to her.

Let's suppose, for the sake of argument, that all other animals lack the cognitive sophistication we ascribe to humans (whatever that sophistication may be) and, in particular, the capacity for rationality. Thus, as one argument goes, because animals lack rational ability, their capacity to suffer is greatly reduced or perhaps non-existent. If we granted this argument, we would have to admit that humans lacking rational ability, such as the severely mentally incapacitated and infants, would also have greatly reduced capacities for suffering, and perhaps these would be non-existent. It is indeed likely that certain forms of suffering are reduced in non-rational beings, be they certain humans or non-humans. For example, a rational being may suffer from fear of losing his job and the inability to provide financial stability for his family. But just as there are forms of suffering that may be greater in rational beings, there may also be forms of suffering that are greater in non-rational beings. A non-rational being cannot rationalize or explain away his suffering. ${ }^{49}$ Consider that an animal captured from the wild does not comprehend what is happening to him and may experience raw terror, even if he is just being implanted with a tracking device for his protection prior to being released. In such cases, animals may experience far more terror and suffering than humans would.

The lack of comprehension and inability to intellectualize their experiences is what often makes animals, not unlike infants and severely mentally incapacitated humans, more vulnerable to certain forms of suffering. Additionally, the pain and/or suffering experienced by a nonrational being may take up the whole of his experience. A common therapeutic method employed to treat prolonged mental suffering or pain in people is cognitive behavioral therapy. This teaches people to override or mitigate their negative experiences by altering their thought patterns and their behavioral responses. Thus humans can be taught to use thought to escape pain and other forms of suffering. The intensity of pain can also be mitigated through expectations, memories and the consideration of, and attention to, other interests and situations. ${ }^{50}$ A non-rational being, on the other hand, may not be able to mentally escape or expect the pain or other negative experience to end and his whole attention may be on that negative experience. ${ }^{51}$ A baby crying out in pain is a tragic thing to most of us precisely because we suspect that his whole awareness and attention at that moment is on his pain and, as a result, he may be suffering tremendously. Similarly, an animal's pain or negative emotional experience (such as terror) may be magnified 
because of the lack of ability to mentally escape it or rationalize it; as a result, that animal may also be suffering tremendously.

Whatever we determine about the cognitive abilities of animals, the presence or absence of certain forms of cognition may not preclude the ability to suffer but only, perhaps, prevent (or conversely increase) the ability to experience certain forms of suffering. Though other animals may lack the complexity of many of the emotional or cognitive capacities possessed by adult humans, this does not imply that their experiences are any less relevant to them. To paraphrase Mark Bekoff, even if a mouse does not have the same sense of 'I-ness' as you or I do, this does not mean that he cannot feel that something is painful or pleasurable to him and cannot suffer or enjoy. ${ }^{52}$

Ultimately, what is relevant here is not how one suffers but the fact that one does suffer. There are indeed differences between humans and other animals, but these differences don't in themselves deny the presence of suffering in animals. The accumulation of evidence certainly demonstrates that animals can feel pain and the evidence is mounting that animals possess a variety of emotional and cognitive abilities. Coupled with their biological and evolutionary continuity with humans, this evidence forms a reasonable basis for assuming that animals can suffer from negative emotional and psychological states, such as distress and fear. Given the evidence, and given the immense harm we may be causing animals by denying or ignoring their suffering, the burden of proof should be on those who maintain that animals do not suffer. If a chicken lives her entire life in a cage so small that she is unable to stretch her wings, an activity that would be normal, the burden of proof should be on those who maintain that we have not caused her to suffer. If there is a possibility that a cat in a laboratory lives in daily terror of what might be done next to him, the burden of proof should be on those who argue that he is not suffering. When taken as a whole, the aforementioned evidence offers a strong case for the existence of animal pain and suffering, and this book will proceed on the premise that the animals discussed experience both and that, therefore, their welfare is relevant.

\section{Other potential objections}

Even if human health advocates acknowledge the ability of animals to suffer, it may still be objected that human health and welfare take priority over animal welfare. This brings us to the second and third main objections that may be argued against including animal welfare 
in public health. There is a common misconception among health professionals that the maximization of human health requires a disregard for, or the minimization of, the welfare of other animals. In other words, there is a perceived conflict between human health and welfare and the welfare of animals. An extension of this position is that we depend on using animals in ways that require that we disregard or minimize their welfare in order to promote human health. That is, animal welfare is not only irrelevant but in opposition to human health. For example, some may argue that factory farming is the most efficient and economical method of producing enough food for and preventing undernourishment in the growing human population. But, as is argued in Chapters 4 and 5 , not only does factory farming threaten our health by contaminating our environment and generating infectious diseases, we can actually feed more people by reducing our use of animals as food.

Perhaps the example most frequently cited in support of minimizing the welfare of animals is animal experimentation. Experimenting on animals is a highly controversial issue. Advocates of this practice frequently employ the fallacy of a false dilemma: that we must choose to care about human suffering or about animal suffering, and that we cannot do both. This erroneous thinking leads us to believe that we must either experiment on a mouse (or a dog or a monkey ...) or we must experiment on a human child, implying that we are forced to make a choice-it's the animals or us. A hypothetical moral question frequently posed to advocates of animal protection is the sinking boat scenario. If you were on a sinking boat with a child and a dog, the scenario asks, and you could save only one, which would you save: the dog or the child? While this seems like an interesting moral dilemma (and one that is frequently used to force the reader to weigh the worth of other individuals, such as their spouse or their child), public health does not have to choose between the dog and the child.

Public health can cheat this scenario altogether. We generally have a third option: one that provides a way out of this polarizing quagmire, in which an advocate for humans can also be an advocate for animals, and in which these two roles are complementary. Although there will always be times when the welfare of an animal will be pitted against the welfare of a human (or humans), how we decide to resolve such issues should be done in a manner that causes the least amount of harm possible to all involved, human and animal alike. As this book will demonstrate, however, in most cases where humans interact with animals, not only can we help people without harming animals, we can also best help people by not harming animals. We will explore numerous 
examples illustrating that our treatment of animals is integral to many of the causes of, and potential solutions to, some of the biggest human health threats. In other words, not only can we save the dog and the child, but in order to save the child we have to save the dog. Our mental and physical health, the state of our environment, the safety of our food, and the efficacy and safety of our medical treatments are inextricably tied to how we choose to treat other animals.

\section{A very brief history of public health}

The final potential significant objection to the premise of this text is that issues of welfare, protection and ethics concerning our use of, and interaction with, animals are outside the purview of public health and medicine. Sociologists, animal advocates, philosophers and others, it could be argued, are better situated to deal with such social issues. The idea of including the welfare of animals in the sphere of public health may be seen as a radical one. However, public health is no stranger to social concerns (or radical ideas). On the contrary, public health, throughout its history, has been an integral part of social change. A brief look at the evolution of public health over the past two centuries reveals its expanding role in social issues.

Public health has frequently challenged, directly and indirectly, cultural mores and societal prejudices. At a time when illnesses were often blamed on the lifestyles of the poor, Dr John Snow and clergyman Henry Whitehead marched into the slums of London and identified the root of a merciless cholera epidemic sweeping across the city. ${ }^{53}$ They proved that the cause of the 1854 epidemic was not the 'moral failings' of the lower classes, which was thought to make them more susceptible to cholera, but water contaminated by sewage. ${ }^{54}$ The idea that contaminated water could be the source of an epidemic was a radical one at the time and was contrary to the predominant 'miasma theory', which contended that spreading of foul-smelling odors, mostly by the city's laborers, was the root cause. ${ }^{55}$ Once the connection between sanitation and illnesses such as cholera was revealed, sanitation campaigns dominated public health practice in nineteenth-century Europe and North America. It became evident to health professionals that infectious diseases in the Industrial Age were rooted not only in increased industrialization but also in impoverished living conditions and inequities in social standing. ${ }^{56}$ The 1854 cholera epidemic spread rapidly throughout London's labor population because of their dismal and crowded living conditions. The sanitation campaigns reflected an awareness of 
the powerful connection between people's social position, their living conditions and their health outcomes. ${ }^{57}$

German pathologist Rudolph Virchow, credited as being the 'father' of modern pathology and who greatly advanced public health, asked, 'Do we not always find diseases of the populace traceable to defects in society ${ }^{\prime 58}$ Understanding the connection between social standing and health, he became a social justice activist on behalf of the poor. ${ }^{59}$ It became widely acknowledged that infectious diseases could not be successfully combated until the underlying social causes were unraveled and tackled, and the sanitation campaigns transformed health care professionals into social activists. ${ }^{60}$

Since the sanitation campaigns, as new health concerns arose, the social roots of illness and health became increasingly evident and activism by health professionals grew dramatically. During the early twentieth century, public health expanded to address maternal and infant mortality and child undernourishment. ${ }^{61}$ High rates of occupational disease and industrial injuries led to programs for industrial hygiene and occupational health. ${ }^{62}$ Work-related health problems, such as coal workers' black lung disease and silicosis, led to campaigns for safer work environments. ${ }^{63}$ Advocacy on behalf of women, children and workers for basic rights and protections, such as improved working conditions, limitations on working hours and restriction or banning of child labor practices, was an important extension of public health and medical practice.

Many social revolutionaries in public health emerged. Nurse Margaret Sanger was a rebel in her time. In the early twentieth century, she witnessed countless women dying during childbirth from unwanted pregnancies. ${ }^{64}$ She challenged the long-held doctrine that prevention of conception was 'indecent and disgusting' ${ }^{65}$ Sanger became a fugitive of the law when she responded to the needs of destitute mothers by opening family planning clinics for the poor in New York City and advocating for women's rights to access to contraception and control over their own fertility. ${ }^{66}$

Since Sanger's time, women's and children's rights campaigns have led to the prioritization of child health care, improved reproductive health care for women, access to family planning and the emergence of public health programs, such as the Women's, Infants, and Children food service. By the latter half of the twentieth century, increasing awareness within psychiatric and patient communities resulted in collaborative efforts to tackle long-held stigmas against the mentally ill. ${ }^{67}$ As these stigmas began to diminish, the promotion of mental health, in addition 
to physical health, was incorporated for the first time into routine public health policy. In 1946, the World Health Organization (WHO) astutely defined health as 'a state of complete physical, mental and social wellbeing and not merely the absence of disease or infirmity' ${ }^{68}$ Since its inception, WHO had also recognized the link between health and social structure, justice and social disparities. The founders shared a vision of health as pre-eminently shaped by social conditions. ${ }^{69}$

Over the course of the twentieth century, public health steadily increased its scope. Evolving needs and societal values led to substantial changes and the inclusion of ethics as a field in public health. Following the revelation of Nazi experimentation on war camp prisoners, and the aftermath of the infamous Tuskegee experiments in which AfricanAmerican men with syphilis were denied access to penicillin in order for researchers to study the course of the illness, significant regulations were created for the protection of human subjects in research. ${ }^{70}$ Ethics became an integral discipline within public health throughout the world.

The HIV/AIDS pandemic that began in the 1980s is arguably the single biggest health crisis to transform public health policy and practice. ${ }^{71}$ The pandemic drew attention to the plight of some of the most vulnerable groups and the need to prevent discrimination in law and health practice. ${ }^{72}$ When much of the world vilified homosexuals, public health workers zeroed in on this population as being in need of priority protection. The marginalization of and discrimination against gays, lesbians and bisexuals, the stigma that followed those infected with HIV and the disregard for their welfare led to a rather revolutionary approach to health. Jonathan Mann, then head of WHO's global AIDS program, led the call to acknowledge the vital linkage between health and human rights. ${ }^{73}$ He recognized that HIV infection rates were closely connected to inequality, injustice, discrimination and the failure of public health to recognize the roots of vulnerability worldwide. ${ }^{74}$ Through collaborative efforts with human rights activists, public health eventually recognized that the fundamental protection of human rights is one of the most effective means to ensure positive conditions for health. Public health also acknowledged that there is a moral obligation to protect and become advocates on behalf of the most vulnerable populations throughout its policies and practice.

As these examples over the past two centuries demonstrate, public health has come to recognize how social factors influence our health and, as societal values have changed, so too has the practice of public 
health. Public health now encompasses a vast scope of social and non-social factors, which were previously not recognized as having an impact on health. Ethics has become an integral arm of public health. Topics such as women's autonomy and reproductive rights in religious fundamentalist countries, health disparities in developed nations, poverty and disadvantaged populations in developing nations, the equitable distribution of medications and disparate burdens placed on minority groups now predominate public health discussions. Over the past two centuries public health has been challenged to go 'beyond its traditional work to embrace programs to decrease poverty, illiteracy, environmental degradation, and violence' ${ }^{75}$ Individual rights; discrimination based on gender, ethnicity, sexual orientation and age; political, legal and economic forces; poverty, war and violence; personal behaviors; the structure of urban environments and numerous other factors impact our health.

Alvin Tarlov, Director of the Texas Institute for Society and Health, explained that 'there are five major categories of influence on health: genes and associated biology; health behaviors, such as dietary habits, tobacco, alcohol and drug use, and physical fitness; medical care and public health services; the ecology of all living things; and social and societal characteristics' ${ }^{76}$ As stated by Stephen Leeder from the Menzies Centre for Health Policy in New South Wales, Australia, 'the new public health makes considerable reference to sociological and anthropological insights and engages with the world of human behavior ... in pursuit of better health'. ${ }^{77}$ As a result of these insights, physicians, nurses and public health practitioners have become an integral part and are, at times, at the forefront of social change. We now understand that how we interact with and treat each other, how we view others, how we share (or do not share) our resources, how we eat, how we work, how we play, how we shelter ourselves, how we think, how we govern ourselves, how we spend money and how we relate to our environment-in short, how we live-influence our health. Despite all that we have come to understand, however, we have yet to fully appreciate one of the major categories described by Tarlov: the ecology of all living things. It is true that we study in great detail the life cycle of mosquitoes and are applying greater attention to environmental changes. But we have always overlooked (with the few exceptions described earlier), and continue to overlook, a significant facet of human existence that has prevailed since our beginning: our relationship with and treatment of other animals. 


\section{The elephant in the room, her welfare and why that matters to our health}

Today, food security issues, limited health care resources, unsustainable consumption patterns, environmental degradation, bioterrorism, global warming, human population growth, obesity, novel infectious diseases, world hunger and violence are now emerging as the most urgent public health issues that we face. ${ }^{78}$ The complexity and multifactorial roots of these issues necessitate a public health strategy that goes well beyond the health sector. We have started to do this. Due to recent and significant changes in our climate, for example, the fields of public health and medicine have begun to acknowledge that how we treat our planet affects our health. To fully tackle these urgent issues head on, we must also acknowledge that many of them are inevitably linked with how we treat other animals. It is overly simplistic and inaccurate to say that every human-animal encounter that is connected with human illness is a result of our actions or is avoidable. Yet, a significant proportion of these connections are, at least in part, a result, directly or indirectly, of our disregard for or minimization of the welfare of animals.

If public health is concerned about the public's health, then it must address a series of issues that it has so far largely avoided — and that affect the welfare of animals. If public health is concerned with how climate change endangers human health, it should also be concerned about factory farming's impact on global warming. If public health is concerned about the threat of new and deadly infectious diseases, it should also be concerned about the wildlife trade's potential to unleash a pandemic worse than HIV/AIDS. If public health is concerned about limited health care resources, it should also be concerned about how a significant portion of our health care dollars is diverted into animal experiments of dubious medical value. If public health is concerned about the alarming rise in antibiotic resistance, it should also be concerned about the ubiquitous feeding of antimicrobials to animals on industrial farms. If public health is concerned about violence, it should also be concerned about the connection between violence toward animals and violence toward people. If public health is concerned about the epidemic of obesity, it should also be concerned about obesity's connection to our unprecedented consumption of animal products. If public health is concerned about the safety of our food, it should also be concerned about factory farming's effect not just on the safety of animal food products but also 
on that of our fruits and vegetables. In short, if public health is concerned about the protection and promotion of human health, and if public health acknowledges that every other facet of human existence plays a role in our health, it must also acknowledge that how we relate to animals is a major determinant of our health. If public health is concerned about public health, we must turn our attention to the elephant (and every other animal) in the room.

Public health must now recognize that when we disregard the welfare of animals and cause them to suffer, we also disregard our own welfare and cause our own suffering. Today, humans are harming animals on a record scale. Worldwide, over 64 billion land animals are raised and slaughtered for food annually. ${ }^{79}$ Most of them are now reared in massive factory farms, a source of immense animal suffering. ${ }^{80}$ Annually, more than 115 million animals suffer as they are used in experiments or to supply the biomedical industry throughout the world. ${ }^{81}$ Every year, billions of animals are ripped from their natural habitats and sold as pets and entertainers, or killed for their fur, skin, meat or other body parts. And, with our own population explosion, we are destroying habitats for other animals at a rate that might soon become irreversible.

Despite the rise in our maltreatment of certain animals, we are seeing an unprecedented increase in the number of humans sharing their lives with other animals solely for the purpose of companionship. This is a drastic change from much of human history in which our interactions with animals revolved mostly around our dependence on them for food, transportation, labor and similar uses. In the USA, for example, 62 percent of households now include companion animals. ${ }^{82}$ Increasingly, couples, empty nesters and professionals are living with companion animals in lieu of having children. ${ }^{83}$ Companion animals are now commonly viewed as integral family members. We see them as our kids. We lavish them with attention. We invest in their health and welfare. We mourn their deaths. And, we spend lots and lots of money on them. Americans now spend about $\$ 41$ billion a year caring for companion animals. The companion animal industry is one of the fastest growing in the USA, and similar market increases are occurring in other parts of the world. ${ }^{84}$

We are increasingly attached to the animals with whom we share our lives but at the same time have become increasingly detached from other animals, such as those we consume for food. The result is our inadvertent (or deliberate) complicity in the suffering and slaughter of billions of animals each year. Our contradictory relationship with 
animals is nothing new. Historically, we have tended to view certain animals as 'pets' worthy of our regard and others as 'pests', whose welfare we have discounted. Animals with whom we are less familiar, who may be less aesthetically pleasing to us or who have been used as food or tools have always carried the brunt of our maltreatment. But what is different-and allows for unprecedented amounts of suffering-in more recent times is this: animal mistreatment is hidden from the public eye. What happens to animals in factory farms, in experimental laboratories, in circuses and other 'entertainment' venues, in fur farms, in individual homes and during transport across the globe usually takes place behind closed doors.

Slowly, this suffering is coming to light. Daily, news sources cover animal welfare issues and the public is increasingly expressing outrage against abuses of animals. Public perception of animals is starting to change-in part because of our growing relationship with and understanding of some animals, and in part because of the scientific evidence exploring animal minds. Animal protection has gained ground and is now a significant social movement. A wide range of academics and professionals, including philosophers, ethicists, legal and religious scholars, authors, sociologists and physicians, have joined the movement. As a result, laws are being passed worldwide to confer greater protection for animals in many circumstances. For instance, in 2009, voters in the state of California banned the use of battery cages for egg-laying hens and of veal and sow gestation crates, following the lead of four other states. The entire European Union (EU) will ban battery cages in 2012. ${ }^{85}$ In 2010, the Spanish region of Catalonia made headline news by becoming the first mainland region of Spain to ban bullfighting, a centuries-old tradition. For the first time in its history, China, in 2009, unveiled legislation to address deliberate cruelty to animals. ${ }^{86}$ Norwegian law has included fish in its Animal Welfare Act since 1974. Since 1997, The EU officially recognized animals as being sentient, able to feel pain and experience emotions, and this was written into their basic treatise. The legally binding protocol requires the EU and its member states to 'pay full regard to the welfare requirements of animals' as 'sentient beings' ${ }^{87} \mathrm{~A}$ record 91 animal protection laws were passed in the USA in 2008, eclipsing the previous record of 86 new laws in $2007 .{ }^{88}$

Discourse about the use of animals in experimentation and alternatives to their use is becoming of greater interest, and the practice of eating vegan diets-diets that exclude all animal products-is a growing worldwide phenomenon. There are around 1500 books on animal ethics today and at least 12 academic journals in the field of animal 
ethics. ${ }^{89}$ In the USA, animal law is one of the fastest growing fields in legal scholarship. ${ }^{90}$ The study of human-animal relations is a burgeoning interdisciplinary field in sociology, psychology and zoology on both sides of the Atlantic. ${ }^{91}$ Worldwide, there is increasing recognition that animals have inherent value and there is growing skepticism over the long-standing belief that the suffering we cause them is morally irrelevant.

Public health must also recognize the welfare of animals. We must pay attention to whether or not the animals we encounter are abused, are forced to endure their lives in confinement, live under daily terror, are deprived of environmental stimulation, are removed from their own kind or are deprived of meaningful social interactions because all of these factors may affect humans. A beaten elephant may become a rampaging elephant; an abused cat may be a stepping stone for someone on their way to abusing a child; a distressed pig in a factory farm may be a reservoir of infectious diseases; and a terrified rat in the laboratory may produce study results that endanger human health.

The chapters that follow will explore these correlations in greater detail. First, it should be stated that, while humans are obviously also animals, most often the term 'animals' will be used rather than 'nonhuman animals' for convenience throughout this book. Second, rather than narrowly being defined by the strictest definition, the term 'public health' is here meant to include all health-related disciplines and professionals, including health care practitioners, public health officials, research scientists and medical ethicists. Third, this book serves as an introduction to animal welfare and public health. It is by no means meant to be an exhaustive account of how every human-non-human relation affects our health. Rather, it is meant to be an overview of some of the major ways in which we encounter and/or use other animals and how those interactions or uses affect human welfare.

Sadly, we have hardly begun to consider the thousands of ways that the human-animal relationship impacts human health. But once the public health profession recognizes the importance of this relationship, it will be able to start finding effective solutions for many of the causes of so much suffering and ill health in both humans and animals. Fortunately, many individual health practitioners are starting to recognize the human-animal welfare connection. Researchers and nurses are increasingly interested in the human-animal bond and how it can benefit human health. Public health agencies, like the American Public Health Association, are asking for a moratorium or ban on intensive animal agriculture in light of its enormous negative environmental impact. 
There are doctors and scientific centers, like the Johns Hopkins Center for Alternatives to Animal Testing, which are finding and promoting superior non-animal testing methods. These first steps, as exciting as they are, only begin to shine the light on the path ahead and on the work yet to be done. In the final chapter, a way forward and a paradigm shift in how public health views the status of and our relationship with other animals is suggested. The future of public health is clearto improve our health we must realize that the welfare of animals is intricately linked to our own. 